\title{
Abbreviations Used in the Dictionary
}

Cap. beginning with a capital letter

Cf. compare

Demon. demonstrative

Eng. of English origin

Fig. figuratively

Lit. literally

Redup. reduplication

Syn. synonym

Var. variant 
